\title{
Search and research during surgical training
}

\section{Saul Goldenberg ${ }^{\mathrm{I}}$}

${ }^{\mathrm{I}}$ Editor-in-Chief

Physicians must deal with patients and must be competent. Must be a good skilled manual worker; a craftsperson. But, it is not sufficient to be a good artisan, It is necessary to have knowledge based in scientific investigation.

The diligent inquiry or examination of data, reports, and observations in a search for facts or principles.

Residents must answer three basic questions regarding diseases:

What is the disease?

How is the disease?

Why the disease?

What is the physiopathology basis of treatment? informations.

It is necessary to search medical literature for scientific

Correct surgical technique essentially requires practice, constant effort and skill. This may be achieved in the experimental laboratory through work in animal models. The fundamental aim of surgical practice applied to animal models is to prepare the surgeon as best is possible. Human beings are not experimentation animals and medical knowledge should not be tested upon them.

Surgical residents must reserve part of the time to experimental surgery. It is also the opportunity to create a vocation for research and to learn the fundamentals of scientific methodology.

Such activities would afford an ideal opportunity on a possible doctoral thesis. It stimulates residents to enter in postgraduate programs.

Teaching staff and surgical instructors should create the scientific spirit necessary to secure patient care, productive research efforts and a wider scope of knowledge and advance in science.

It should be remember that this experimental conception, progressed in the last decades. Animal experimentation and surgical research have contributed to the progress of surgical science. Surgical research is a necessity not only for basic researchers but also for clinical professionals and postgraduates.

In order to hold citizenship values, it is necessary not do anything to the human being that we would not like to have it done towards each one of us.

Researches and technical practice must be performed in the experimental surgery laboratory and the "learning curve" shall follow ethical principles to oneself and to the others.

My personal conviction is to give prominence to the importance of experimental surgery in surgical training. 